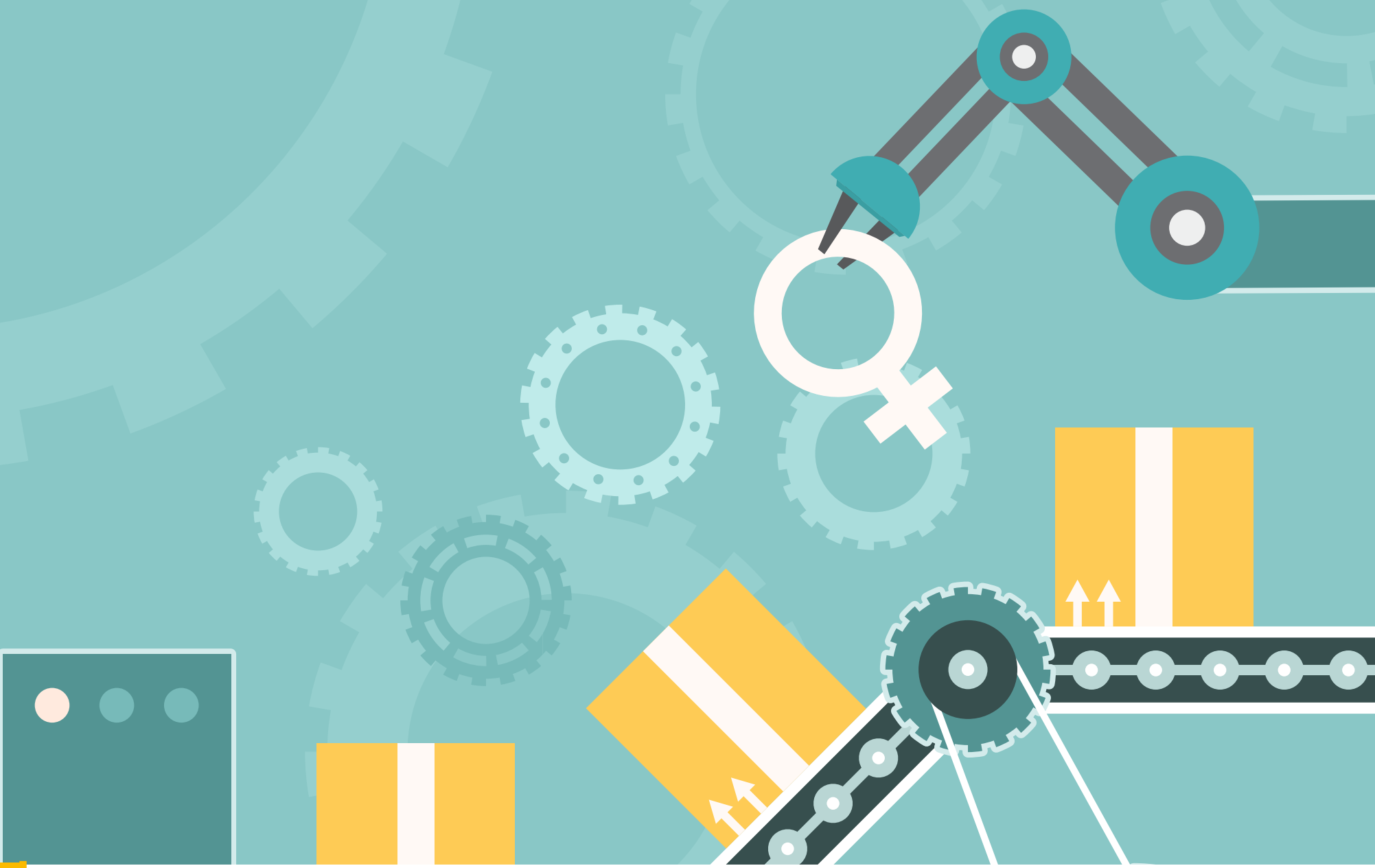

\title{
Mercado laboral y género
} Impacto de la

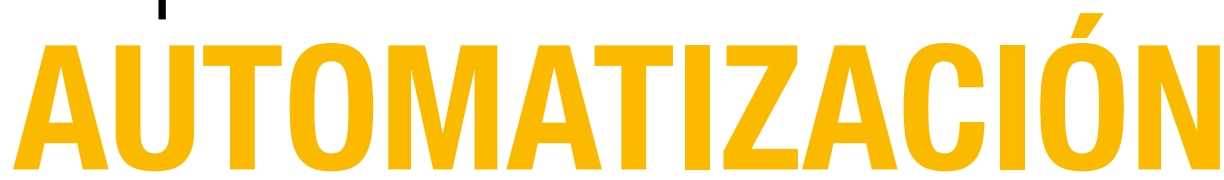

A pesar de que hay muchos titulares en prensa que aseguran que el empleo femenino va a ser el más perjudicado, los escasos estudios que han contrastado este impacto muestran resultados todavía \section{contradictorios.}

Por Goretti Cabaleiro, PhD en Economía de la Empresa y Métodos, Universidad Carlos III de Madrid, España. Académica FEN-UAH.

n la última década, el efecto de la automatización en el mercado laboral ha captado una atención cada vez mayor y ha alarmado a una parte significativa de la población. Sin embargo, las preocupaciones con respecto a los cambios tecnológico no se limitan a la era moderna. En el pasado, dados los rápidos cambios tecnológicos, John Stuart Mill, Marx y
Keynes ya analizaron las posibles consecuencias de la sustitución de la maquinaria por el trabajo humano (Mokyr, 2015). Por ejemplo, en 1900 , aproximadamente el $40 \%$ de los empleados de EEUU trabajaba en granjas, mientras que, en la actualidad, menos del $2 \%$ de las personas lo hacen en los campos; en 1950, alrededor del $24 \%$ de los empleos estaban dedicados a la manufactura; en la actualidad, solo alrededor del 9\% trabaja en esta industria (Rotman, 2018).

Ahora bien, aunque es cierto que no es la primera vez que se discute el tema, los investigadores muestran que los avances actuales son más amplios y rápidos que en períodos anteriores, argumentando que esta vez los efectos adversos no seguirán un crecimiento lineal: será exponencial y dejará a muchas personas sin el tiempo necesario para adaptarse. En ese sentido, el Informe del Instituto Global McKinsey (2017) establece que las consecuencias de la automatización en el mercado laboral actual serán "de una escala sin precedentes".

LauraTyson, Asesora Económica Jefe del ex Presidente Clinton de los EE. UU. y profesora de la Universidad de Berkeley en California, dice: “Esta es la primera vez que vemos que la tecnología podría reducir la demanda de trabajadores humanos" (Equipo editorial 2018).

Daron Acemoglu, investigador del MIT, señala: "Si bien el big data, la automatización y la inteligencia artificial deberían, en teoría, aumentar la productividad de las empresas, impulsar la economía y crear más empleos, la realidad no es así. . . [] Últimamente, por diversas razones, el aspecto destructivo de la tecnología que eli- 
mina puestos de trabajo ha sido más prominente "(Rotman, 2018).

Algunos de los estudios sobre el efecto de la automatización en el mercado laboral que se han citado con frecuencia en prensa son: (i) Frey y Osborne $(2013,2017)$, quienes concluyen que el $47 \%$ de los empleos en EEUU es susceptible de ser automatizado; (ii) los resultados del Mckinsey Global Institute (2017) que estiman que el $51 \%$ de los empleos en los EEUU se automatizará para el 2030; (iii) Nedelkoska y Quintini (2018) quienes concluyen que las personas que trabajan en la industria manufacturera o en el sector agrícola, las mujeres, los trabajadores con menos educación y aquellos quienes trabajan bajo un contrato de aprendizaje tienen más probabilidades de ver sus trabajos automatizados en 32 países; y (iv) un estudio realizado por PricewaterhouseCoopers (2018) que concluye que los trabajadores administrativos o de oficina son los que enfrentan una mayor probabilidad de automatización, a corto y mediano plazo, alcanzando el $49 \%$ para fines de la próxima década. Resultados como estos salen continuamente en la prensa, asustando a la población, generando un deterioro de la salud mental y una mayor adversidad al cambio.

Sin embargo, aunque estas noticias son impactantes, muchos de los resultados que muestra la literatura son contradictorios y a menudo se malinterpretan. Primero, hay que tener en cuenta que los resultados se refieren a la "viabilidad técnica" de la automatización con las tecnologías actuales, pero que la cantidad real de empleos perdidos dependerá de los costos y beneficios de reemplazar a las personas por máquinas. Segundo, los principales estudios relacionados con la automatización se han llevado a cabo en economías avanzadas donde la tecnología se desarrolla $y$, a su vez, es más fácil de implementar. Hay un estudio, por ejemplo, basado en la economía alemana, que tiene muchos más robots que EEUU, donde se demuestra que su introducción no ha tenido un impacto agregado en el empleo (Dauth et al. 2017). Tercero, en la actualidad existen muchos trabajos que combinan tareas rutinarias (fáciles de automatizar) con tareas no rutinarias (difíciles de automatizar) que no pueden desagregarse sin que haya una reducción de la productividad. Por eso, hay autores que defienden que el efecto de la automatización será de complementariedad entre la máquina y el humano en vez de total sustitución (Autor et al. 2015).

Finalmente, los impactos del cambio tecnológico en el mercado laboral dependen no sólo de dónde afecta éste directamente, sino también de cómo se ajustan otras partes de la economía. Por ejemplo, también pueden generarse empleos adicionales a través de la demanda de nuevas tecnologías y mayor competitividad generada.

En cuanto al impacto de la automatización en el mercado de trabajo según el género, a pesar de que hay muchos titulares en prensa que aseguran que el empleo femenino va a ser el más perjudicado, los escasos estudios que han contrastado este impacto muestran resultados todavía contradictorios. Por ejemplo, el informe de la OCDE (2017) concluye que, en promedio para todos los sectores, hombres y mujeres están expuestos al mismo riesgo de ser automatizados. La principal razón es que el riesgo de automatización es mayor en el sector manufacturero, construcción y transporte, donde el empleo masculino domina, y en sectores relacionados con la

\section{"El Informe del Instituto Global McKinsey (2017) establece que las consecuencias de la automatización en el mercado laboral actual serán 'de una escala sin precedentes"'}

alimentación y el retail, donde lo hace el empleo femenino. Ahora bien, este estudio puntualiza que el nivel de educación y las habilidades adquiridas son una herramienta poderosa para reducir el riesgo de automatización ya que menos del $5 \%$ de los trabajadores con un título universitario tienen un alto riesgo de perder su trabajo en comparación con el $40 \%$ de los trabajadores con un título educativo inferior (Arntz, Gregory y Zierahn, 2016; OCDE, 2016a). Por tanto, dado que en promedio en todos los países de la OCDE hay más mujeres que hombres con educación superior (OCDE, 2016b), lo que se desprende de este estudio es que las mujeres se encontrarán en una posición más ventajosa para luchar contra la automatización. En la misma línea, el análisis de Roberts, Lawrence and King (2017), tomando de base el mercado laboral de Reino Unido, sugiere que un $46,8 \%$ de los trabajos desempeñados por hombres versus un $40,9 \%$ de los trabajos desempeñados por mujeres tienen el potencial técnico para ser automatizado. La principal razón que sustenta sus hallazgos es que la inversión en tec-

*Para consultar por referencias comunicarse con la autora del artículo, gcabaleiro@uahurtado.cl nología para aumentar la productividad es menor en los sectores dominados por mujeres, como el cuidado de personas. Ahora bien, este estudio puntualiza que esta menor probabilidad de automatización no quiere decir que las mujeres tendrán buenas condiciones de trabajo, ya que lo que se predice es que éstas van a permanecer en trabajos mal pagados que los empleadores han optado por no automatizar. Además, los autores argumentan que es probable que la introducción de la tecnología aumente los salarios de los trabajadores mejor pagados, que en su mayoría son hombres, lo que lleva a una mayor disparidad salarial. Por lo tanto, concluyen que la automatización podría aumentar la desigualdad de género a menos que tanto las mujeres como los hombres tengan una buena educación y puedan acceder a nuevos empleos bien pagados. Por otro lado, los resultados del estudio del International Monetary Fund, IMF, (2018) indican que, dado el estado actual de la tecnología la probabilidad de automatización es ligeramente mayor para la fuerza laboral femenina: $11 \%$ vs. $9 \%$. Sin embargo, esta probabilidad aumenta para las trabajadoras con menor nivel de educación, de mayor edad (superior a 40 años), y aquellas en puestos de oficina, servicios y ventas. Además, con el objetivo de tener una proyección más detallada en el tiempo, el Informe de PriceWaterHouseCoopers, PwC, (2018) extiende el análisis distinguiendo tres ondas de automatización desde la actualidad hasta 2030. Específicamente, definen: 1) la onda algorítmica, hasta principios de 2020, donde consideran que se realizará la automatización de tareas computacionales simples y el análisis de datos estructurados, afectando básicamente a los sectores impulsados por datos; 2) la ola de aumento, hasta finales de 2020, donde se realizará una interacción dinámica con la tecnología para el apoyo administrativo y la toma de decisiones, y 3) la ola autónoma, hasta mediados de la década de 2030, donde primará la automatización del trabajo físico y la destreza manual y la resolución de problemas dinámicos del mundo real. En términos generales, este informe concluye que mientras el empleo femenino va a ser el más afectado por la automatización en la próxima década, los empleos masculinos van a enfrentar un mayor riesgo de automatización (34\%) que las mujeres $(26 \%)$ en el largo plazo. La lógica detrás de esta cifra es la mayor probabilidad de que el trabajo masculino esté en sectores orientados a tareas manuales, como la manufactura, la construcción, el transporte y almacenamiento mientras que la participación del empleo femenino tiende a estar más concentrada en sectores como la educación, la salud y el trabajo social, que requieren más habilidades personales y sociales y que, tienden a 
ser menos automatizables. No obstante, cuando los autores tienen en consideración el nivel de educación de los trabajadores, se encuentra que los hombres y mujeres con altos niveles de educación tienen tasas de automatización estimadas similares a largo plazo ( $11 \%$ vs $12 \%$, respectivamente), aunque el tipo de ocupación que desarrollen sea diferente: los trabajadores varones con un alto nivel de educación tienen más probabilidades de ser empleados en el sector de la información y las comunicaciones (hombres: 9\% frente a las mujeres: $4 \%$ ), mientras que las mujeres altamente educadas tienen más probabilidades de ser empleados en la educación (mujeres: $29 \%$ frente a hombres: $14 \%$ ). Para los trabajos con un bajo nivel de educación, por ejemplo, hay una diferencia notable entre hombres y mujeres. Los hombres enfrentan un mayor riesgo estimado de automatización (52\%) en comparación con las mujeres con bajo nivel educativo $(29 \%)$ dado el tipo de ocupaciones en los que se desarrollan. Por ejemplo, los trabajadores masculinos con bajo nivel educativo desempeñan tareas artesanales y relacionados, son operadores de maquinaria y ensambladores, mientras que las trabajadoras con bajo nivel educativo se desempeñan

\section{"Dado que en promedio en todos los países de la OCDE hay más mujeres que hombres con educación superior, las mujeres se encontrarán en una posición más ventajosa para luchar contra la automatización"}

lidad y con acceso a pocas prestaciones sociales.

Dados los resultados anteriores se puede concluir lo siguiente. Primero que, aunque la magnitud del efecto de la automatización en el mercado laboral según el género todavía es incierta y presenta resultados contradictorios en términos probabilidad de automatizase reduce cuando el nivel de educación es mercado laboral es relativamente tardía y una de las más bajas de América Latina. Las mujeres representan un 48,5\% de la fuerza laboral (INE, 2018) y se concentran en trabajos de menor calificación y remuneración. Según el informe de CEPAL (2018) las mujeres que acceden a un trabajo remunerado, en promedio, trabajan en peores condiciones que los hombres, en empleos más vulnerables, muchas veces sin contratos y sin acceso a prestaciones sociales (Weller y Roethlisberger, 2011; CEPAL, 2014b, CEPAL, 2016a; como empleadas de servicios y ventas y en ocupaciones elementales, como limpiadoras, cuidadoras y ayudantes.

Finalmente, Hegewisch, Childers and Hartmann (2019), basándose en el mercado laboral estadounidense, concluyen que mientras 7 hombres trabajan en puestos amenazados por la automatización, 10 mujeres lo hacen. Al mismo tiempo, estos autores puntualizan que el trabajo femenino está sobrerepresentado en los puestos con mayor y menor probabilidades de automatización. Es decir, que las mujeres se desempeñan en su mayoría como cajeras, secretarias o contables, con un alto riesgo de automatización, pero también como cuidadoras de niños y de ancianos o educadoras, con un bajo riesgo de automatización. Sin embargo, de nuevo, estos autores puntualizan que estos trabajos "seguros" para las mujeres están relacionados con condiciones a menudo desfavorables: donde se paga menos dado el mismo nivel de educación que otros trabajos, de baja ca-
OIT, 2018a). Por tanto, este nuevo escenario supone un gran desafío. Se necesita una educación actualizada de alta calidad, asesoramiento, aprendizaje permanente y reformas sistemáticas en el sistema educativo. El mercado laboral exige profesionales con habilidades que hasta ahora no eran tan importantes como la capacidad de comunicación, la empatía, el trabajo en equipo, el liderazgo, la resolución de problemas, la autoorganización y las habilidades digitales relacionadas con las tecnologías de la información y las telecomunicaciones. Sin esta inversión en educación, la automatización podría profundizar las diferencias entre géneros todavía más. Por tanto, esperemos que desde ya se empiece ción para que no solo los empresarios se beneficien de una mayor productividad, sino que también los empleados nos beneficiemos de una menor jornada laboral, una mayor conciliación familiar y una mejor salud mental. $\mathbf{E}$ a planificar una transición hacia la automatiza- onviene observar primero que es difícil juzgar la conveniencia del uso de SMs de manera independiente del funcionamiento y de la regulación del mercado eléctrico en su conjunto. ¿Por qué? Porque el advenimiento y la necesidad de instalarlos es solo una parte de una discusión mucho más amplia que se conoce como smart grid management -o manejo de redes inteligentes. La idea y el objetivo esencial detrás de este concepto es el diseño (dada la rápida aparición de nuevas tecnologías) de una infraestructura más eficiente y confiable tanto en la transmisión como en la distribución de electricidad. Resulta entonces apropiado estudiar en este contexto algunos de los principios básicos que lideran la discusión pública.

Los medidores tradicionales son aún el medio más usado para medir el consumo de energía de las familias y comercios. Estos registran el consumo en kW horas de manera acumulada durante un período de tiempo, usualmente 30 o 60 días. Por otra parte, un SM es un tipo de contador avanzado de electricidad que calcula el consumo (o producción) de una forma más detallada que los medidores tradicionales.

En otras palabras, los SMs registran el consumo de energía en tiempo real.

Un SM es solo el dispositivo instalado en su empresa, comercio u hogar. Lo importante es la función que este desempeña en un sistema de medición inteligente (SMI, de ahora en más). Pero ¿qué es un SMI?

La Comunidad Europea en su nota interpretativa 2009/72/EC afirma que un SMI es "un sistema que permite la comunicación bilateral entre el consumidor $y$ el oferente $u$ operador con el objetivo de promover servicios que faciliten el uso eficiente de la energía en el hogar.

Así, en este sistema, el SM es el dispositivo que facilita la comunicación entre usuarios y empresas.

\section{LA EXPERIENCIA INTERNACIONAL}

Los SMI llegaron para quedarse. El despliegue de los SMs está creciendo de manera exponencial alrededor del mundo. Strother y Lockhart predicen, por ejemplo, que hacia el 2022 se habrán instalados más de mil millones en todo el mundo. En la Unión Europea la normativa imperante exige que hacia el 2020 , el $80 \%$ de las familias lo tengan instalados en sus hogares. Para el 2022, el principal mercado de redes inteligentes será China con una tasa de despliegue de SMs de alrededor del $70 \%$ de todos los hogares. En EEUU existe un crecimiento sostenido de la instalación de SMs, especialmente en es- 